\title{
Operational efficiency of maneuver coordination rules for an airborne separation assurance system
}

\author{
R. Schild, J.K. Kuchar \\ Department of Aeronautics and Astronautics \\ Massachusetts Institute of Technology \\ Cambridge, MA USA
}

\begin{abstract}
To facilitate the continuing growth of air traffic and the request for more operational flexibility, users will require various systems not only to prevent collisions but also to allow on-board separation assurance without air traffic controller intervention. This responsibility shift implies that maneuver coordination between aircraft will no longer lie with one central controller but rather will be based on maneuver coordination rules typical to fully or partly decentralized systems. Clearly, higher rule complexity, assuming a proper design, leads to additional gains in safety and maneuver efficiency for particular operational environments. However higher rule complexity often requires more input information and more computation for rule application, which might have a negative impact on safety and increases system cost. This paper discusses the rule complexity / rule efficiency problem. Rule efficiency measures are discussed and mathematically defined. Further, an approach to evaluate different rule structures for an airborne separation environment using computerbased models is described and results are presented.
\end{abstract}

\section{Introduction}

The air traffic conflict detection and resolution process consists of several tasks to ensure separation or avoid collisions depending on the scope of the system (Figure 1) [1].

The first task is to check the environment for potential conflicts with the help of state and possibly velocity vector information. Based on the information available, future positions can then be estimated.

Conflict detection is based on the estimation of future vehicle positions and through the application of predefined metrics on the situation in order to decide whether or not a conflict is present. This metric may include a sole parameter (e.g., distance) or may be a combination of several parameters (e.g., distance, time and maneuvering cost).

After the detection of a conflict, a conflict resolution phase requires appropriate maneuver action and information distribution to all aircraft involved in the conflict.

Under certain operational conditions, rule systems such as Visual Flight Rules (VFR) [2] in aviation or Collision Avoidance Rules for shipping [3] are used for the resolution maneuver coordination. These rules define priorities to vehicles involved in the conflict and suggest a corresponding resolution maneuver. Explicit maneuver coordination is possible but not necessarily required. Envisioned self separation environments such as "Free Flight" [4] will likely need some form of resolution maneuver coordination.

In a decentralized system, each agent has to coordinate decisions with other agents involved in the conflict. Predictability of decisions and resolution maneuvers is essential to maintain an orderly flow of traffic and to prevent collisions. Rule systems for maneuver coordination (priority determination \& resolution maneuver) would facilitate this predictability.

The complexity of maneuver coordination rule systems depends on the operational environment and 
its characteristics. In today's existing rule-based systems quite simple rule sets are used and work well (e.g. VFR). Responsibility for decisions rely with the human operator (for legal reasons), who has to understand the underlying principles. The use of too complex rule systems holds the potential for human misinterpretation while they might not necessarily increase operational system performance.

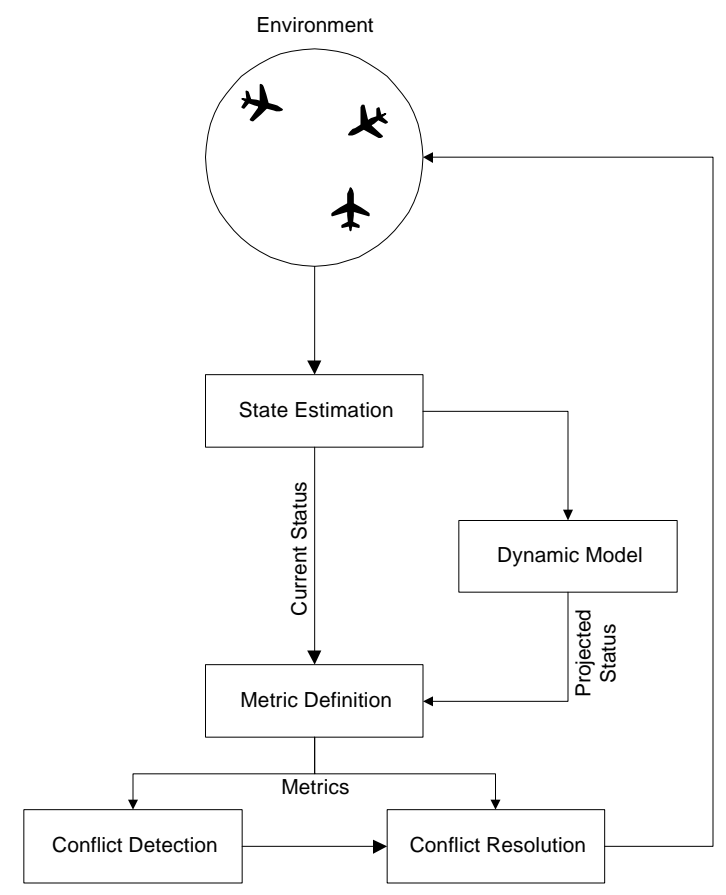
Figure 1: Generic Conflict Detection and Resolution
Process

Additionally, the increase of rule complexity follows the law of diminishing returns, meaning that the additional gain through more input information and higher rule complexity is getting smaller. Finally, increasing rule complexity may make it more difficult for human operators to understand the basis behind conflict decisions, potentially resulting in nonconformance and distrust of the system.

\section{Evaluation of rule systems}

New system designs are generally tested upon whether specific predefined criteria, e.g. operational needs, are met or not. Careful attention has to be placed on the definition of these criteria. In the case of evaluating different designs of maneuver coordination rules, criteria such as " ... route efficiency, time efficiency, fuel efficiency and other practical aspects related to displaying and executing the resolutions" can be used. [5]

For the particular evaluation scheme several processes may be considered:

- A system evaluation can be done through a team of proven experts. Most often the system operators are involved. In the evaluation process of the Extended Rule of the Air (EFR) proposed by Eurocontrol [6] experts from fields such as piloting, ATC operation, and aircraft dynamics and equipment have been involved.

- A computer based simulation (i.e. a fast-time simulation with no human operator involved) would be a more complex but more objective evaluation method. It is certainly less biased to well established beliefs within the operator or expert community and offers the advantage of being able to objectively run through far more scenarios and options in significantly less time. Further, if necessary, advanced methods to optimize the design can be applied. However the analytical criteria used in a simulation must reflect operational needs as closely as possible and it is often difficult to formulate those criteria (i.e. if cognitive aspects are critical). Additionally, it is difficult to model human behavior in fast-time simulations beyond a simple level. often it is assumed that humans will act exactly as the rules dictate when in reality there may be cases in which this is not so.

- And finally a human-in-the-loop computer based simulation can be a means to allow cost efficient but quite realistic evaluation (compared to flight trials with real aircraft) of even complex cognitive aspects of designs. However the number of scenarios subject to test are limited due to time-constraints related to the duration of a human-in-the-loop simulation run and the availability of suitable human test subjects.

\section{Rule Design}

\subsection{Information needed for Maneuver Coordination}

For maneuver coordination based on rules essentially three different kinds of information can be used:

- position or state information

- velocity vector information 
- additional (intent) information, e.g. emergency status, target altitude, next waypoint

All information listed above is envisioned available in an ADS-B message. The available information pieces can be used solely or in combination. Table 1 lists examples of maneuver coordination rules used in today's operational environment or those proposed in the literature with the corresponding information used in the priority determination process. The column labeled "evaluated" indicates whether this information was used for priority determination rules in this paper.

The most basic information about conflicting aircraft are their positions (either absolute or relative). Priority determination in simple coordination rules such as VFR are entirely based on state information (i.e. the other vehicle's relative position).

\begin{tabular}{|c|c|c|}
\hline Information & Example & evaluated \\
\hline \multirow[t]{3}{*}{ State } & Visual Flight Rules (VFR) & $\checkmark$ \\
\hline & \begin{tabular}{|l|} 
ATLAS Autonomous \\
Flight Rules [6]
\end{tabular} & $\checkmark$ \\
\hline & $\begin{array}{ll}\text { IFR/VFR Flight } \\
\text { Level Rule [2] } \\
\text { NLR's Altitude for } \\
\text { Direction-of-Flight } \\
\text { (AFDOF) [5] }\end{array}$ & $\begin{array}{l}\checkmark \\
\text { (partly) }\end{array}$ \\
\hline $\begin{array}{l}\text { State and } \\
\text { Velocity } \\
\text { Vector }\end{array}$ & $\begin{array}{l}\text { Distance to closest point } \\
\text { of approach - EFR rules } \\
\text { used in FREER [6] }\end{array}$ & \\
\hline $\begin{array}{l}\text { State, } \\
\text { Velocity } \\
\text { Vector and } \\
\text { Flight Phase }\end{array}$ & $\begin{array}{ll}- & \text { Maneuverability/Avai } \\
\text { lability - EFR rules } \\
\text { used in FREER [6] } \\
\text { - } \\
\text { Phase-of-Flight } \\
\text { Priority (PFP) - NLR } \\
\text { Free Flight Trials [5] }\end{array}$ & $\sqrt{ }$ \\
\hline
\end{tabular}

Table 1: Information used for maneuver coordination with examples

The velocity vector could also be used to determine priority based on the vertical rates of the aircraft involved. Considering that aircraft fly at constant altitudes in cruise, a vertically moving aircraft could be assigned lower priority than level cruising aircraft. This would only require the knowledge of the vertical rate.

Further state information and the velocity vector can be used to determine priority. The proposed EFR rules use a reliable estimation of the distance to the closest point of approach (CPA) based on current states and the explicit knowledge of the velocity vector of the aircraft involved in the conflict. In case both aircraft are in the same (sub-) flight phase the aircraft farther away from CPA, i.e. the faster aircraft, will have to give way to the slower aircraft.

Even more additional information (priority categories such as emergencies or ambulance flights) possibly in conjunction with state and/or the velocity vector can be used to determine the flight phase of particular aircraft and to assign priority between conflicting aircraft based on it.

\subsection{Resolution Dimensions}

Initiating a resolution maneuver requires at least one aircraft to change its flight path, i.e. to change its velocity vector. Three directions are possible:

- The speed of the aircraft (length of the velocity vector) can be increased or decreased;

- A turn can be initiated, i.e. a change of the velocity vector in the horizontal plane;

- The vertical rate can be changed, i.e. change of the velocity vector in the vertical plane.

Changes of the velocity vector are possible as a single-state maneuver (e.g. change of the speed only) or in combinations (e.g. speed and heading). Combinations of maneuvers can be performed simultaneously or in sequence. Further, maneuver strength can be shared among conflicting aircraft or not (e.g., Eby's potential field model used in NLR Free-Flight trials) (see table 2).

Table 2 below lists some potential resolution options possible and examples if known. As in table 1 the columns labeled "evaluated" indicates whether the resolution option was evaluated in the computer simulation discussed here.

\begin{tabular}{|l|l|l|}
\hline Order & Example & evaluated \\
\hline $\begin{array}{l}\text { Shared } \\
\text { Resolution; } \\
\text { Only one change } \\
\text { of the velocity } \\
\text { vector }\end{array}$ & $\begin{array}{l}\text { NLR repulsive } \\
\text { force principle } \\
\text { (with sole } \\
\text { maneuver) [5] }\end{array}$ & $\checkmark$ (partly) \\
& $\begin{array}{l}\text { NLR Extended } \\
\text { VFR Overtaking } \\
\text { Rules (EVOR) [5] } \\
\text { ATLAS head-on } \\
\text { encounter [6] }\end{array}$ & \\
\hline $\begin{array}{l}\text { Shared } \\
\text { Resolution; }\end{array}$ & - & $\checkmark$ (partly) \\
Sequential & & \\
Changes of & & \\
Velocity Vector & & \\
\hline
\end{tabular}




\begin{tabular}{|l|l|l|}
\hline $\begin{array}{l}\text { (e.g. level off } \\
\text { followed by a turn }\end{array}$ & & \\
\hline $\begin{array}{l}\text { Not Shared } \\
\text { Resolution; } \\
\text { Only one change } \\
\text { of the velocity } \\
\text { vector }\end{array}$ & $\bullet \quad \begin{array}{l}\text { Visual Flight } \\
\text { Rules [2] }\end{array}$ & $\checkmark$ \\
\hline $\begin{array}{l}\text { Not Shared } \\
\text { Resolution; } \\
\text { Sequential } \\
\text { Changes of } \\
\text { Velocity Vector } \\
\text { (e.g. level off } \\
\text { followed by a turn }\end{array}$ & - & \\
\hline
\end{tabular}

Table 2: Conflict resolution options with examples

\section{Rule Evaluation Criteria}

System evaluation, beside many other aspects requires careful selection of the proper evaluation criteria. These have to be chosen according to the operational requirements and constraints of the environment where the system will be used.

For aircraft separation purposes definitely the most important operational constraint for maneuver coordination rules is that proper application ensures safe separation of all aircraft at all times. This constraint can be considered a hard constraint, disqualifying those rules not meeting it.

Between rules meeting the constraint of ensuring safe separation, however, differences in operational efficiency of different rules should be taken into account. In an effort to evaluate rule sets a cost function was used [7]. Beside other parameters the cost function is based on cost for additional fuel and time, required by resolution maneuvers. However one disadvantage of this approach is the requirement of detailed information about different parameters, e.g. cost indexes, fuel flow depending on the aircraft mass, which might vary between users and equipment. Another approach using only cross-track distance from the initial planned route instead of fuel and time reflects the operational efficiencies in an appropriate way and is far easier to model. The latter approach was chosen for this study.

As a third evaluation criterion, the number of maneuvers necessary to achieve safe separation was chosen. Free Flight Studies involving pilots showed that pilots tend to dislike the necessity of several resolution maneuvers to achieve safe separation [8].
Pilots requested to keep the number of resolution maneuvers low. Therefore the number of maneuvers resulting from the application of coordination and resolution rules was inserted into the evaluation function.

In summary, three criteria are used in this study:

- safe separation

- cross-track distance from the initial planned route

- number of resolution maneuvers

The criteria are mathematically combined giving the following evaluation value $(\mathrm{EV})$ function:

$$
E V=\frac{k}{\text { Offset } \cdot \text { Maneuver }^{2}+\text { Penalty }}
$$

Offset (in kilometers) is the average of the maximum cross-track distances from the initial planned route required by a (combination of) resolution maneuver(s). Maneuver is defined as average number of changes of the velocity vector to resolve a conflict. The square of maneuver is used to emphasize a low number of maneuvers. A Penalty of 100 is added if a the required separation is not met. The penalty is needed for the genetic algorithm to prevent reproduction of rule combinations not meeting separation minima. The parameter $k$ was chosen to be 10 to provide convenient magnitudes for $\mathrm{EV}$, though any value could be used.

The EV function defines operational efficiency and is used to compare different rule combinations. The higher the EV value the higher the achieved operational efficiency.

\section{Rule Evaluation}

For rule evaluation a fast-time flight simulation was developed. The simulation integrates:

- equations of motion allowing the calculation of flight paths for point mass aircraft;

- aircraft performance data of Airbus A340 aircraft based on Eurocontrol's Base of Aircraft Data (BADA) [9];

- a database defining different meta-rules for priority determination and conflict resolution;

- a database with a discrete number of conflict situations;

- the calculation of the evaluation value based on the above specified criteria. 


\subsection{Rule Definition}

Different meta-rules were defined and stored in a database. Meta-rules are based on existing rule systems (e.g. VFR), suggested systems found in the literature or logical combinations of priority determination and resolution maneuver options as described above.

A meta-rule consists of different rules which are combined forming a decision tree. Several rule inputs are connected to a rule output (decision or action). Additionally meta-rule parameters such as sectors (see figure 2) or a maneuver strength (bank angle or vertical rate) are variable and are subject to optimization in the evaluation process.

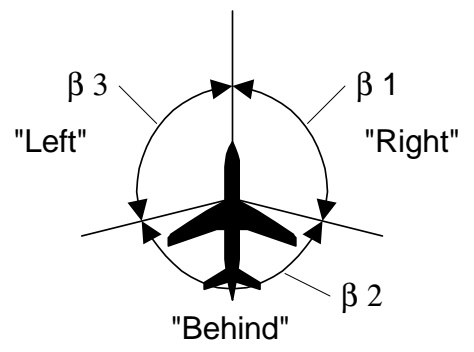

Figure 2: Parameters $\beta_{1}, \beta_{2}$ and $\beta_{3}$ for Sector Definition in a meta-rule

The simulation is linked to an optimization system using genetic algorithms [10]. The EV function is used as the objective function for the genetic algorithm optimizing parameters of meta-rule combinations for comparability. In the meta-rules parameters such as the angles $\beta_{1}, \beta_{2}$ and $\beta_{3}$ which describe sectors around the aircraft in the horizontal plane (as used in VFR) are variable (see figure 2). Varying parameters within a meta-rule result in different operational efficiencies.

For evaluation purposes different combinations of meta-rules were tried. Each combination should yield the best evaluation values possible for a predefined, discrete number of conflict situations. Thus optimization of meta-rule parameters is necessary. Genetic algorithms were found to be suitable for this task.

Eleven meta-rules, four for priority determination and seven for conflict resolution, were defined and evaluated. Tables 1 and 2 show which rule designs were used. Table 3 provides explanations about the meta-rules used. The seven conflict resolution metarules organized, according to the necessary changes of the velocity vector, in three groups. In the simulations the resolution meta-rules in the specific groups were combined with the meta-rules for priority determination and parameters were optimized using genetic algorithms.

\begin{tabular}{|c|c|}
\hline Meta Rules & Explanation \\
\hline \multicolumn{2}{|c|}{ Priority Determination } \\
\hline VFR & $\begin{array}{l}\text { Models the priority decision part of } \\
\text { existing VFR rules. The sector } \\
\text { geometry }\left(\beta_{1}, \quad \beta_{2} \text { and } \beta_{3}\right) \text { is } \\
\text { constant. }\end{array}$ \\
\hline State & $\begin{array}{l}\text { Like VFR but the sector geometry } \\
\text { is used for priority determination. } \\
\text { The sector geometry }\left(\beta_{1}, \beta_{2} \text { and } \beta_{3}\right) \\
\text { is variable. }\end{array}$ \\
\hline State \& Vector & $\begin{array}{l}\text { Like State but aircraft having a } \\
\text { vertical rate unequal zero have } \\
\text { lower priority than aircraft with a } \\
\text { vertical rate equal zero. }\end{array}$ \\
\hline $\begin{array}{l}\text { State \& Vector } \\
\text { \& Flight Phase }\end{array}$ & $\begin{array}{l}\text { Like State \& Vector but the } \\
\text { velocity vector is used to } \\
\text { differentiate between climb, cruise } \\
\text { and descend. Cruise has the highest } \\
\text { priority. Climb has a higher } \\
\text { priority than a Descent. }\end{array}$ \\
\hline \multicolumn{2}{|c|}{ Conflict Resolution } \\
\hline $\begin{array}{l}\text { Maneuver } \\
\text { Lateral }\end{array}$ & $\begin{array}{l}\text { Resolution Maneuvers are } \\
\text { performed in the lateral plane only. } \\
\text { Two meta-rules are used. } \\
\text { - Turn in the direction to pass } \\
\text { behind the conflicting aircraft. } \\
\text { - Always turn to the right } \\
\text { (corresponding resolution } \\
\text { maneuver to VFR). }\end{array}$ \\
\hline $\begin{array}{l}\text { Maneuver } \\
\text { Lateral \& Level } \\
\text { Off }\end{array}$ & $\begin{array}{l}\text { Sequential changes of the speed } \\
\text { vector in the lateral and vertical } \\
\text { plane. Changes in vertical plane are } \\
\text { in the form of a temporary level-off } \\
\text { or a decrease of the vertical rate. } \\
\text { Five meta-rules are in this group }\end{array}$ \\
\hline $\begin{array}{l}\text { Maneuver } \\
\text { Lateral } \\
\text { Vertical }\end{array}$ & $\begin{array}{l}\text { Sequential changes of the speed } \\
\text { vector in the lateral and vertical } \\
\text { plane are possible. Possible } \\
\text { velocity vector changes in the } \\
\text { vertical plane include a level-off, a } \\
\text { decrease of the vertical rate or a } \\
\text { change of the flight level in case of } \\
\text { cruising. Seven meta-rules are in } \\
\text { this group. }\end{array}$ \\
\hline
\end{tabular}

Table 3: Meta-rules used in the evaluation process 


\subsection{Situations}

The rules were evaluated using 19 different conflict situations varying in geometry (horizontally: head-on, crossing and slow-closure encounters; vertically: combinations of cruise, climb and descent) and flight parameters such as velocity and rate of climb/descend are used. Each scenario was weighted by its likelihood of occurrence, based on data provided by the Eurocontrol ACAS evaluation report [11] and a Boeing report about en route Free Flight simulations in the Cleveland airspace [12].

Modeling situations by continuously varying relative closure angles, vertical rates and turns on a stochastical basis is possible and such trials were conducted. These trials showed no significant evaluation value difference compared to using a discrete number of situations weighted with a likelihood of occurrence. Computational effort however is much higher in the former case.

The 19 different conflict situations were used for three different separation environments varying in horizontal and vertical separation minima:

- 5 nautical miles horizontal and $2000 \mathrm{ft}$ vertical

- 5 nautical miles horizontal and $1000 \mathrm{ft}$ vertical

- 3 nautical miles horizontal and $1000 \mathrm{ft}$ vertical

In the simulations it is assumed that crews would initiate a resolution maneuver following rules between 10 minutes and 5 minutes before reaching the closest point of approach (CPA) in the conflict. The resolution initiation thus was modeled for $600 \mathrm{sec}, 500 \mathrm{sec}, 400 \mathrm{sec}$ and 300sec before reaching the CPA. The resulting evaluation values were then averaged.

For simplification purposes at this stage, the simulations used only pairs of conflicting aircraft.

\subsection{Results}

In the figures 3 to 5 , the rule evaluation results for the three separation environments are plotted. Along the $\mathrm{x}$-axis, the priority determination rules used in the study are shown. The points (combined with lines) mark the different combinations of priority determination and resolution maneuver rule options. The evaluation value (EV) of the simulation is plotted along the y-axis. Higher evaluation value (EV) indicates better operational rule efficiency according the criteria specified above.

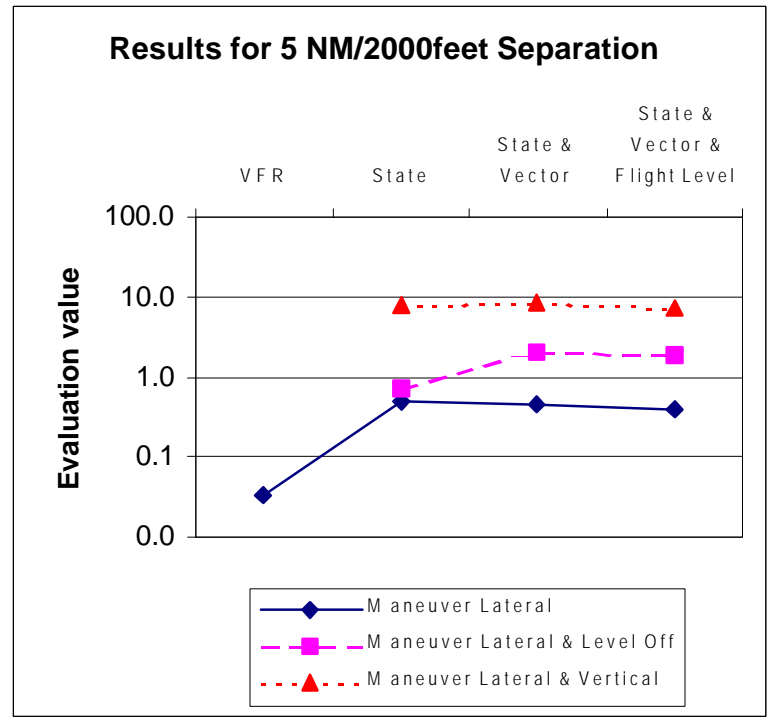

Figure 3: Evaluation results for a separation requirement of 5 NM laterally and 2000 feet vertically

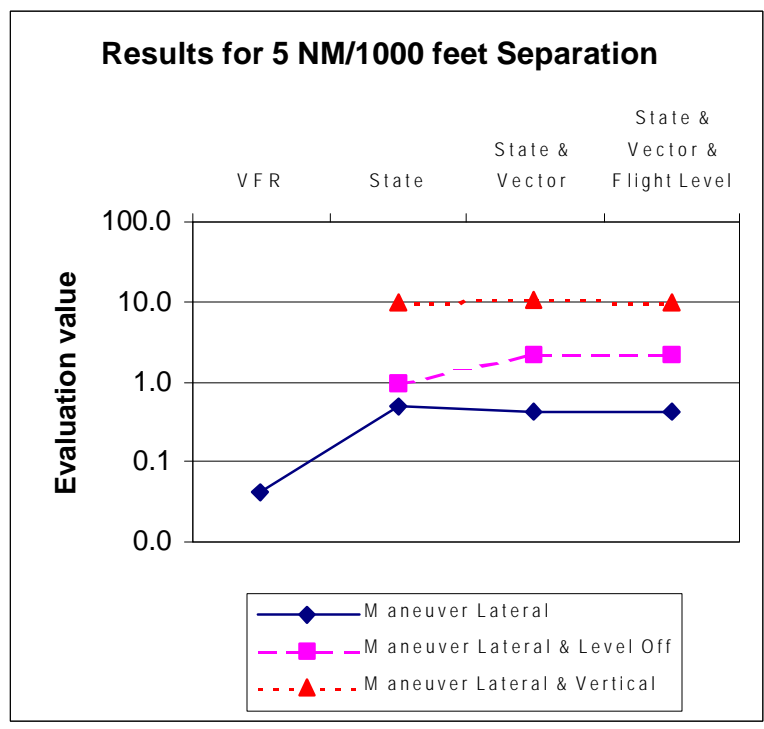

Figure 4: Evaluation results for a separation requirement of $5 \mathrm{NM}$ laterally and 1000 feet vertically

The solid line shows different priority rule efficiencies if only lateral changes of the flight paths are applied for resolution.

The broken line shows priority rule efficiencies if lateral and vertical direction changes are applied solely or in sequence. The simulation showed that for aircraft which have to give way and are either in a climb or descent, modifying the vertical rate is an efficient option to resolve the conflict. 
The dotted line shows priority rule efficiencies if meta-rules include lateral maneuvers, level off and flight level changes. Adapting the vertical rate is the best option for aircraft in climb or descent. For aircraft in cruise, because of the lower vertical separation required, changing the altitude results in a higher EV value than a heading change.

Resolution maneuver rules show a general trend to use vertical direction changes (i.e. reduced rate of climb/descent or changing altitude), which can be explained through the large separation standard ratio between horizontal and vertical and to the form of the evaluation function that was used. Without considering passenger comfort, engine wear or other aspects, changes of the flight path in the vertical direction is more efficient than lateral flight path changes.

Visual Flight Rules (VFR) are used as a baseline in all three scenarios. However the application of VFR violated separation requirements of $5 \mathrm{NM} / 2000 \mathrm{ft}$ and $5 \mathrm{NM} / 1000 \mathrm{ft}$ in cases where the resolution maneuver was initiated at a smaller time to closest point of approach limit (e.g. 300sec) in slow-closure encounter situations. The separation violation, because of the penalty, results in a very low EV value (see figure 3 and 4). With the lower required separation 3NM/1000ft, the VFR evaluation yields a more comparable $\mathrm{EV}$ value (see figure 5).

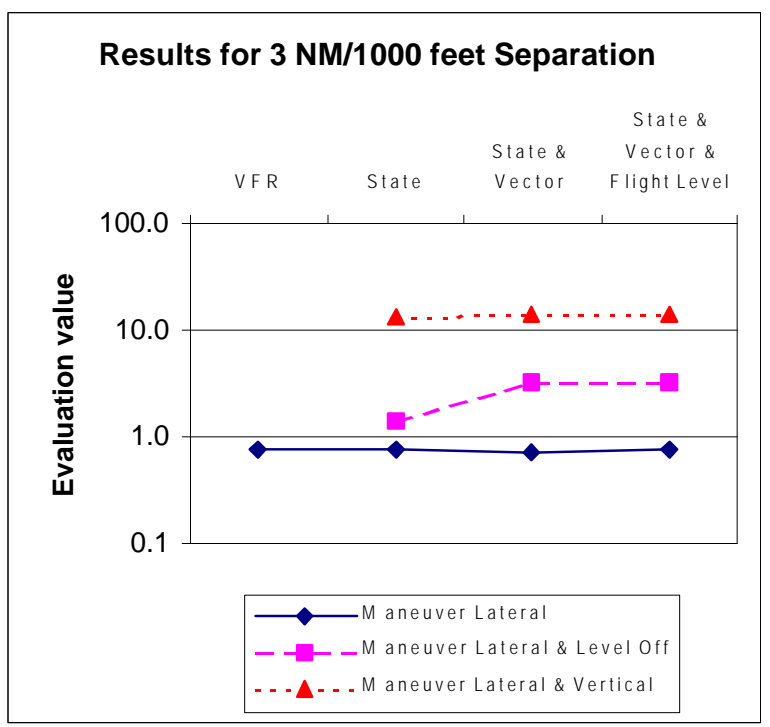

Figure 5: Evaluation results for a separation requirement of 3 NM laterally and 1000 feet vertically

Figures 3 to 5 show that the use of additional information in the priority determination process does not necessarily lead to better results in terms of efficiency. The only major increase in efficiency due to rule complexity across all separation standards was in the Maneuver Lateral \& Level Off line (broken line). An increase of the $\mathrm{EV}$ value occurs if the velocity vector is used for priority determination and maneuvers in the vertical direction are allowed. Using additional information such as the differentiation between flight phases doesn't result in an increase of rule efficiency for the particular situations modeled.

The average number of maneuvers used to resolve conflicts was generally close to 1 . The simulations showed a slight but consistent increase of the average number of maneuvers if rules suggested resolution maneuvers in the vertical direction. The overall evaluation value however is getting higher. The reason once again lies in the different distances for horizontal and vertical separation.

\section{Human Factors and Rules}

Despite the use of a rule structure, the human decision-makers in the cockpit and on the ground will form decisions based on their own internal logic. When a human disagrees with rule-based logic, the outcome may range from a delay in action to a chronic loss of trust in the rules. Mismatches between human decisions and the rule structure may occur in three general areas:

- Observable information. The human may not be able to observe the same information that is used by the rule base, and vice versa. For example, the human will likely not be able to estimate range, bearing, or altitude of another aircraft as accurately or rapidly as an automated rule-base system. Accordingly, the human may disagree with the automation as far as the proper course of action due to this mismatch in the perceived values of the inputs to the decision. Similarly, the human generally has access to more information regarding an encounter situation than is used in the rule base. This may include voice communications that indicate the intentions of another aircraft, weather, etc. Then, a rule-based decision may be clearly unacceptable due to a conflict with this other information.

- Differences in decision-making logic. Humans are very adept at making complex judgments that may be traceable to some form of rule structure, yet there are also many elements of human decision-making that cannot be represented formally by a set of rules. Because humans do 
not naturally follow a rigid series of rules when making a decision, they may also have difficulty fully understanding or predicting the behavior of air traffic in a complex rule environment.

- Differences in the degrees of freedom of action. A rule base is limited in the scope of actions or outputs that it can provide. This may involve restrictions to only lateral maneuvers, for example. The human may consider other options, though the human may also be limited as far as the complexity of action is concerned. A complex rule-based suggestion may not be as well accepted as a simple, single heading vector, for example, solely due to the desire of the human to limit complexity.

The result of these potential areas of conflict is that human performance will likely place constraints on the design of a rule base used for traffic management. Just because a certain rule base is shown to enhance traffic separation in ideal simulations does not necessarily mean that the same rule base would be acceptable to the pilot or controller. Thus, the results presented in this paper need to also be considered in the light of how they may impact interactions between the humans and the rule structure. To be the most effective, the rules should provide for a flexible, rather than restrictive, structure that can adapt to the full range of situations that may unfold during operation.

\section{Conclusions}

The results of the rule evaluation using a fast-time simulation show that higher rule complexity, assuming a proper design, can lead to additional gains in safety and maneuver efficiency. In the simulations, a genetic algorithm is used to optimize rule parameters. Additional study is required to determine whether the rule generated by the genetic algorithm are indeed optimal, or if other solutions may be viable. Also the proper form of an evaluation function is open to debate and would likely change from the one used here to better reflect actual operating costs, safety levels, and human preferences.

The additional gain in rule efficiency through higher rule complexity appears to be driven mainly by the allowance of vertical maneuvers, which, because of separation standards, are more efficient than lateral maneuvers.

The results also suggest that the most efficient use of rules occurs when the information used in the rules is matched with the information used in the resolution maneuvers. For example, there is little if any benefit to adding the vertical vector in the rules if only horizontal maneuvers are allowed for resolution. Similarly, there is a significant benefit to including the vertical vector when using vertical resolution maneuvers.

Explicit human interaction in the form of human-inthe-loop simulation was not investigated. But it is important to note that that human acceptance of rules to solve traffic conflicts is influenced by the complexity of rules and their flexibility of application related to the operational environment.

As air traffic management transitions to a more flexible and efficient mode of operation, it will be necessary to maintain some degree of structure and predictability of traffic through the use of rules. These rules play a similar role as a centralized controller by ensuring coordination and consistency between aircraft actions. The proper selection of rules will be critical to balance complexity, effectiveness, flexibility, equipment requirements, failure robustness, and human acceptance. The study described here represents an initial evaluation of only one component of this important area, and further research by the community is recommended.

\section{The authors}

Raoul Schild has worked as a postdoctoral associate at the Department of Aeronautics and Astronautics at Massachusetts Institute of Technology and now holds a research affiliate position. Additionally he works with Dr. Schild \& Partner as a consultant specializing in air traffic management systems, corporate modeling and decision support systems. He has worked on projects for the airspace arm of DaimlerChrysler Inc., the Deutsche Bahn Group (rail network), Deutsche Telekom AG (telecommunications), Austrian Airlines $\mathrm{AG}$ and Trans World Airlines Inc. (airlines). He received a master degree in mechanical engineering and a Ph.D. in mathematics from Vienna Technical University. $\mathrm{He}$ is a private pilot and teaches navigation courses at a flight school in Austria.

James K. Kuchar is an Assistant Professor of Aeronautics and Astronautics at MIT, where he has been on the faculty since 1995 . His research interests focus on safety-critical decision aiding and alerting systems, advanced cockpit displays, air traffic 
control, safety assessment, and flight simulation. His work on alerting systems won the RTCA William E. Jackson Award and the Council for University Transportation Centers' Wootan/Pikarsky Award in 1995. In research for NASA, he has performed safety assessments for instrument approaches to closelyspaced parallel runways and worked on traffic conflict detection and resolution systems. He has also been investigating policy issues related to air traffic and space launch operations. Dr. Kuchar received his S.B., S.M., and Ph.D. in Aeronautics and Astronautics from MIT, where his work focused on terrain displays for transport aircraft. He is a member of the American Institute of Aeronautics and Astronautics and is a private pilot. At MIT, Dr. Kuchar currently teaches a freshman seminar on back-of-the-envelope engineering, and graduate courses on Flight Simulation and Decision Aiding and Alerting Systems.

\section{References}

1. Kuchar James K. , Yang Lee C., Survey of Conflict Detection and Resolution Methods, AIAA Guidance, Navigation and Control Conference, New Orleans, Aug. 97

2. Mensen H., Moderne Flugsicherung, Springer Verlag, Berlin, 1993

3. Paul Wolfgang, Seeverkehrsrecht, DSV Verlag, 1995

4. RTCA Task Force 3, Final Report of RTCA Task Force 3: Free Flight Implementation,October 1995

5. Gent R.N.H.W van, Hoekstra J.M., Ruigrok

R.C.J. Free Flight with Airborne Separation

Assurance, NLR report

(http://www.nlr.nl/public/hosted-sites/freeflightatm/overview.html), 1997

6. V. Duong, E. Hoffman, L. Floc'hic, J.P. Nicolaon, Capt. A. Bossu (Air France), Extended Rules-Of-The Air To Apply To The Resolution Of Encounters In Autonomous Airborne Separation, EUROCONTROL Experimental Centre, EEC Paris Bretigny, 1996

7. R. Schild, Rule Optimization for Airborne Aircraft Separation, PhD Thesis, Vienna Technical University, 1998

8. Lozito Sandra, McGann Alison, Mackintosh Margreth-Anne, Cashion Patricia, Free Flight and Self Separation from the Flight Deck Perspective, Eurocontrol/FAA ATM Seminar 1997, Paris, 1997
9. Bos A., User Manual for the Base of Aircraft Data (BADA), Revision 2.6, Eurocontrol, EEC Note No.23/97, September 1997

10. Palisade Corporation, Evolver User Reference Handbook, Evolver Version 4.0 - Professional Edition, 1998

11. Hager G., European ACAS Operational Evaluation - Final Report, Eurocontrol EEC Report No. 316, July 1997

12. Warren A., A Methodology And Initial Results Specifying Requirements For Free Flight Transitions, Eurocontrol/FAA ATM Seminar 1997, Paris, 1997 